
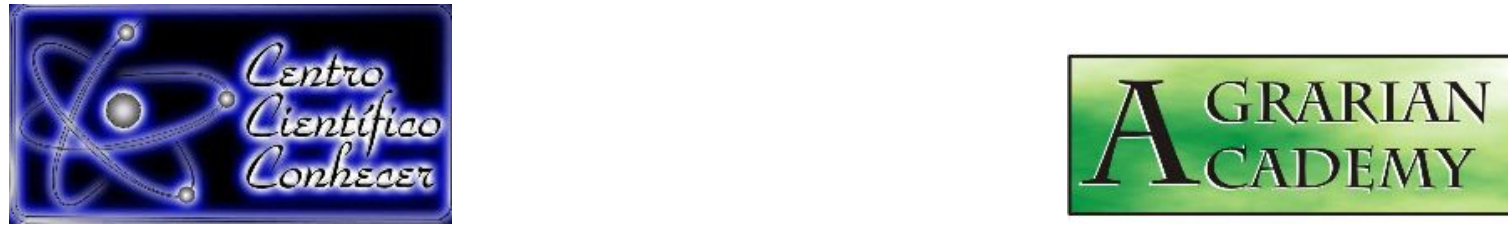

\title{
RESILIÊNCIA DE ESPÉCIES FLORESTAIS DE MATA ATLÂNTICA PARA RESTAURAÇÃO FLORESTAL EM LAGO ARTIFICIAL
}

\footnotetext{
Liliane Garcia da Silva ${ }^{1}$, Carolina Souza dos Santos ${ }^{2}$, João Marcos Pereira Bezerra ${ }^{2}$, Leandra Brune de Almeida Moura ${ }^{2}$, Matheus Neiva de Oliveira Setaro ${ }^{2}$

${ }^{1}$ Professora no Instituto Federal de Educação, Ciências e Tecnologia do Tocantins (IFTO), Paraíso do Tocantins-Tocantins-Brasil (lilianegarcia@ifto.edu.br)

${ }^{2}$ Técnicos em Meio Ambiente do Colégio Técnico da Universidade Federal Rural do Rio de Janeiro (CTUR)
}

\section{Recebido em: 14/07/2018 - Aprovado em: 28/07/2018 - Publicado em: 31/07/2018 DOI: 10.18677/Agrarian_Academy_2018a30}

\begin{abstract}
RESUMO
A reconstrução das condições físicas, ajuste químico do solo e da água; e manipulação biológica, incluindo a reintrodução de espécies da flora e fauna nativas é um desafio para restauração florestal no entorno de lagos artificiais. Nesse sentido o trabalho analisou estabelecimento inicial de espécies endêmicas da Mata Atlântica no entorno de lago artificial, visando restauração desse ambiente. O estudo foi realizado no Colégio Técnico da Universidade Federal do Rio de Janeiro (CTUR/UFRRJ), em Seropédica-RJ, no período de julho a setembro de 2017, utilizando delineamento em blocos ao acaso, com cinco (5) tratamentos (espécies), em quatro (4) períodos (0, 30, 60 e 90 dias), com 4 repetições. A investigação verificou que a espécie Caesalpinia echinata (Pau Brasil) apresentou desempenho, positivo quanto ao crescimento em altura $(\mathrm{cm})$, o Pterogyne nitens (Amendoim Bravo) no caule e a espécie Schizolobium parahyba (Guapuruvú), não recuperou no número de folhas após 90 dias de plantio. Recomenda-se a continuidade do monitoramento e manutenção do plantio em até 2 anos, e o enriquecimento com outras espécies florestais.
\end{abstract}

PALAVRAS-CHAVE: Floresta, hotspot; impactos; revegetação.

\section{RESILIENCE OF ATLANTIC FOREST FOREST SPECIES FOR FOREST RESTORATION IN ARTIFICIAL LAKE}

\begin{abstract}
The reconstruction of physical conditions, chemical adjustment of soil and water; and biological manipulation, including the reintroduction of native flora and fauna species, is a challenge for forest restoration around artificial lakes. In this sense the work analyzed the initial establishment of endemic species of the Atlantic Forest in the surroundings of artificial lake, aiming at restoration of this environment. The study was conducted at the Technical College of the Federal University of Rio de Janeiro (CTUR/UFRRJ), in Seropédica-RJ, from July to September 2017, using a randomized block design with five treatments (species), in four (4) periods $(0,30,60$
\end{abstract}


and 90 days), with 4 replicates. The research showed that the species Caesalpinia echinata (Pau Brasil) presented a growth performance in height $(\mathrm{cm})$, Pterogyne nitens (Braised Peanut) on the stem and the species Schizolobium parahyba (Guapuruvú), did not recover in the number of leaves after 90 days of planting. It is recommended that planting monitoring and maintenance be continued in up to 2 years, and enrichment with other forest species.

KEYWORDS: Forest, hotspot; impacts; revegetation.

\section{INTRODUÇÃO}

Estudos sobre desmatamento em remanescentes florestais da Mata Atlântica do Brasil, e especificamente no estado do Rio de Janeiro, demonstra que essa taxa ocorre em pequenas proporções (Fundação SOS Mata Atlântica \& INPE, 2016), especialmente substituídas frequentemente pelas atividades de agropecuária e expansão urbana (FREITAS et al., 2013). E que para os órgãos de conservação da natureza a sua regeneração florestal é atualmente considerada como uma das maiores prioridades na manutenção desse ecossistema (RIBEIRO et al., 2015).

Aliado às necessidades humanas, lagos artificiais surgem, dos quais são empegados para os usos múltiplos das águas. Sendo que seu entorno, são considerados áreas de preservação permanentes (APPs) por Brasil (2012), pois apresentam valor ecológico e social, embora protegida pela legislação, essas podem apresentar processos impactantes, que derivam dos usos indevidos nas margens recém-formadas (ALARCON et al., 2015).

Assim, esses ambientes degradados demandam de restauração, e para simular um sistema natural ecologicamente integrado com a paisagem, deve-se observar a reconstrução das condições físicas, ajuste químico do solo e da água; e manipulação biológica por reintrodução de espécies (SILVA, 2008). A recuperação natural é demorada e, requer intervenção antrópica, devido ao baixo banco de propágulos e fertilidade do solo (RODRIGUES et al., 2014).

Por isso, que produção de mudas de espécies arbóreas nativas, para reflorestamento ou recomposição de áreas degradadas, é considerada como uma alternativa tecnológica, pois tem finalidade minimizar os impactos, e melhorar as condições edafoclimáticas e a conservação da biodiversidade (LEITE et al., 2017). Onde, devem-se associar espécies com distintos estágios sucessionais, pois estas interagem entre si (LELES et al., 2011).

Para tanto, deve-se observar a escolha de espécies rústicas para se desenvolverem em ambientes hostis e, preferencialmente, com características que contribuam para o reequilíbrio e a estabilização do ecossistema, propiciando a atuação dos mecanismos e processos de colonização, e integração ambiental (FARIAS et al., 2017). Por isso, as ações recuperadoras pressupõem o uso de medidas de proteção do solo, dentre as quais a formação de uma vegetação de cobertura apresenta destaque (RONDON, 2012).

Assim, o presente trabalho investigou o estabelecimento inicial (90 dias) de espécies florestais no entorno de lago artificial, visando à restauração desse ambiente de Mata Atlântica no município de Seropédica, no estado do Rio de Janeiro-Brasil.

\section{MATERIAL E MÉTODOS}

O estudo foi desenvolvido durante no período de julho à setembro do ano de 2017, no entorno do lago artificial, o qual foi originado para dispor águas pluviais da 
drenagem do Colégio Técnico da Universidade Federal do Rio de Janeiro (CTUR/UFRRJ) no ano de 2014, sendo localizado pelas coordenadas geográficas $22^{\circ} 46^{\prime} 41^{\prime \prime}$ sul e $43^{\circ} 40^{\prime} 79 "$ oeste, em Seropédica no estado do Rio de Janeiro, Brasil (Figura 1).

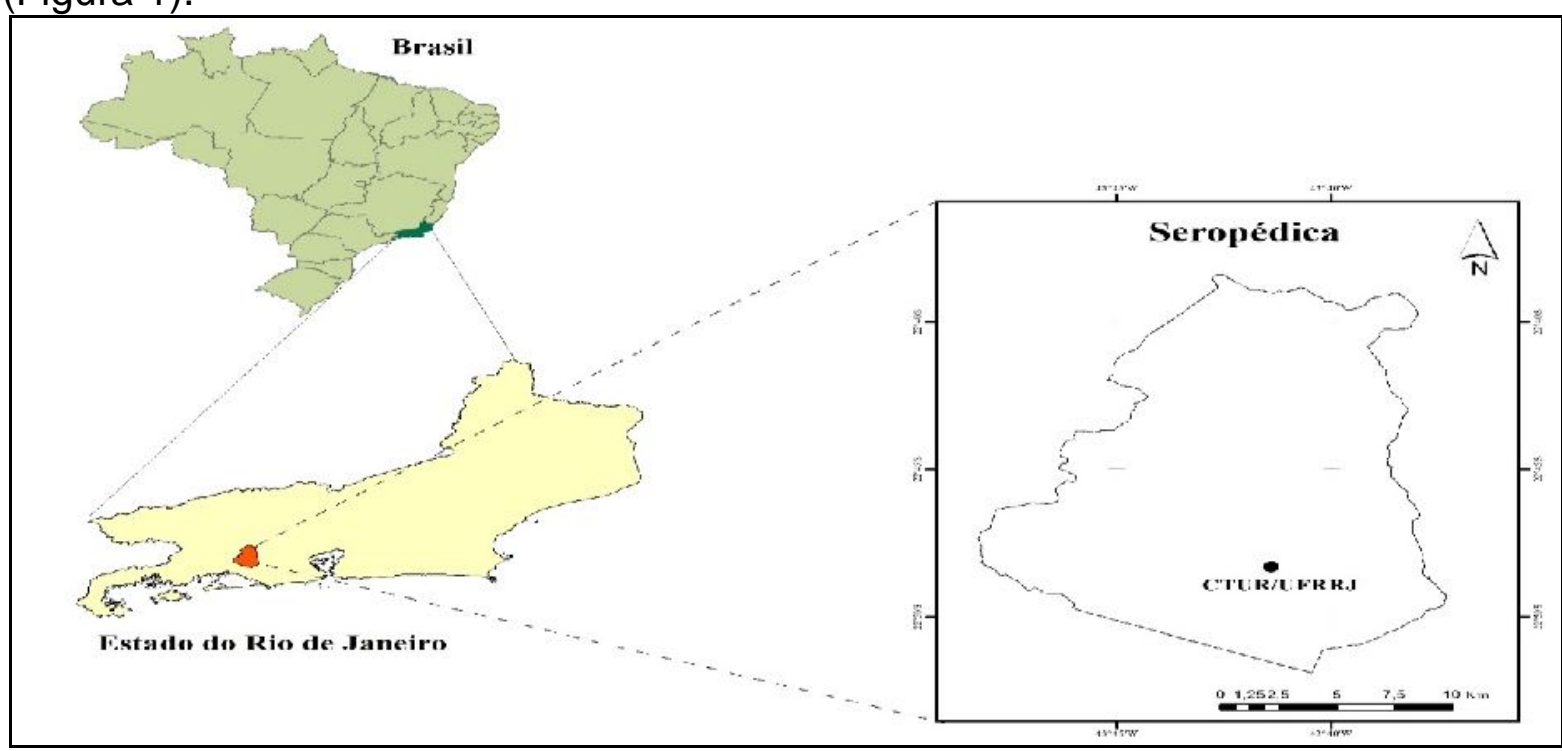

FIGURA 1. Localização da área de estudo, Seropédica-RJ.

A área de estudo está inserida na macrobacia do Rio Paraíba do Sul e na sub-bacia do Rio Guandu. Apresenta ainda um relevo predominantemente suave com declividade máxima de $15 \%$. A pedologia apresenta domínio de Planossolo Háplico, com horizonte superficial arenoso, formado pela aluviação de argila, horizonte Glei em profundidade variada, caracterizado pela textura mais argilosa. $O$ clima é do tipo Aw ou Tropical do Brasil Central, com $23,8^{\circ} \mathrm{C}$ de temperatura média anual, o período seco nos meses de junho-agosto, e chuvoso de dezembrofevereiro.

Para o plantio, foram utilizadas as espécies do Quadro 1, com espaçamento de $4 \times 4$ metros, com covas com 0,5 metros de profundidade, sendo adicionados substrato orgânico e calcário dolomítico, cujo plantio foi realizado em junho de 2017.

QUADRO 1. Espécies florestais utilizadas na restauração florestal no entorno do lago artificial no CTUR/UFRRJ, Seropédica-RJ.

Nome científico Nome vulgar

Cariniana legalis (Mart.) Kuntze

Caesalpinia leiostachya

Caesalpinia echinata

Bauhinia forficata

Schizolobium parahyba

Tabebuia roseo-alba

Couroupita guianensis

Pterogyne nitens

Triplaris americana

Enterolobium contorstisiliquum (Vell.) Morong Inga edulis

Anadenanthera colubrina

Tabebuia chrysotricha

Caesalpinia pluviosa

\begin{tabular}{cc} 
Nome vulgar & Estágio sucessional \\
\hline Jequitibá-rosa & Climax \\
Pau-ferro & Clímax \\
Pau-brasil & Clímax \\
Pata-de-vaca & Secundária inicial \\
Guapuruvú & Secundária inicial \\
Ipê-branco & Clímax \\
Abricó de macaco & Secundária tardia \\
Amendoim-bravo & Secundária inicial \\
Pau-formiga & Secundária inicial \\
Orelha-de-negro & Secundária inicial \\
Ingá & Secundária tardia \\
Angico & Clímax \\
Ipê-amarelo: & Secundária inicial \\
Sibipiruna & Secundária tardia \\
\hline
\end{tabular}

Sobre a área de plantio (Figura 2) foi realizado um experimento em delineamento experimental em blocos ao acaso com cinco tratamentos (espécies), 
em quatro períodos $(0,30,60$ e 90 dias), com quatro repetições, totalizando em 20 parcelas experimentais (Quadro 2). Os parâmetros biométricos analisados foram: altura $(\mathrm{cm})$, diâmetro $(\mathrm{cm})$ e número de folhas (unidade), conforme estudos de Leles et al. (2011).

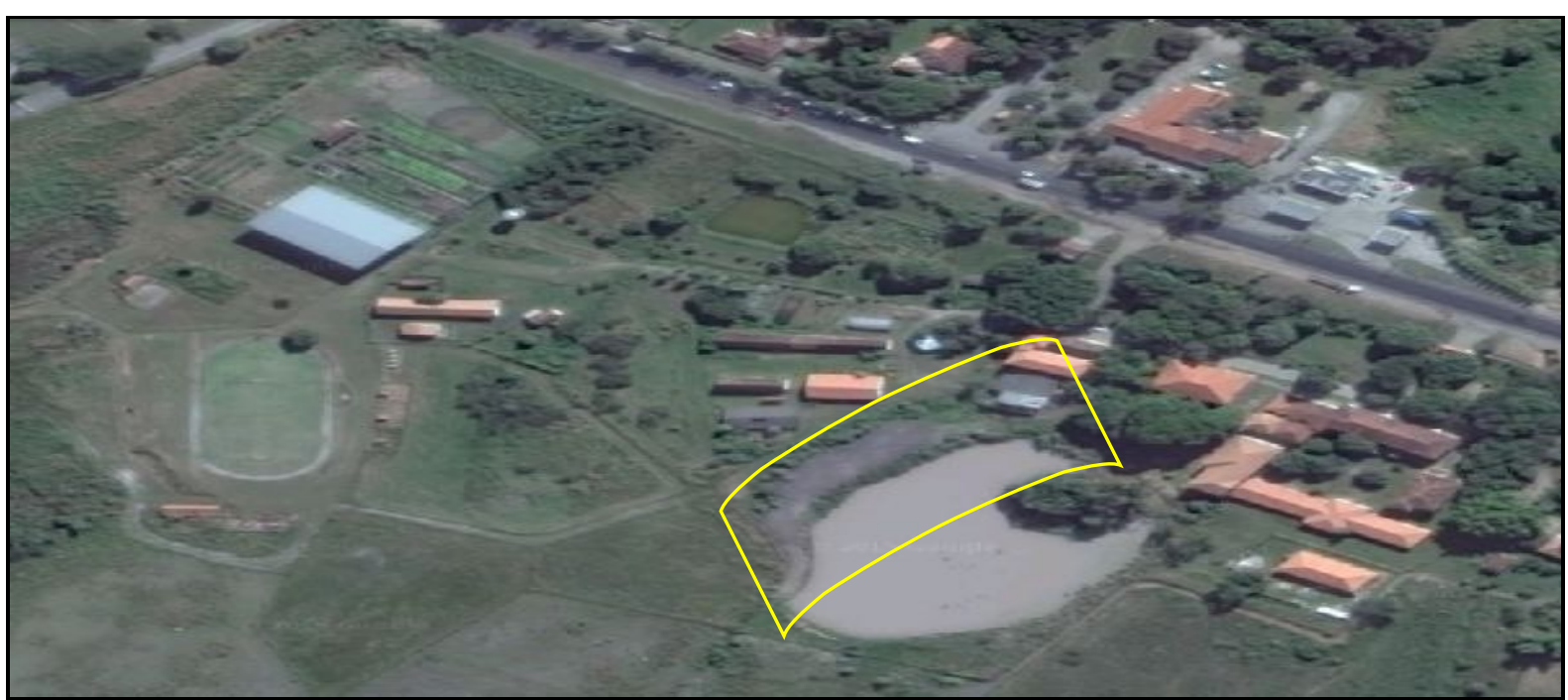

FIGURA 2. Local do plantio das espécies florestais no entorno do lado no CTUR/UFRRJ, Seropédica-RJ (Fonte: Google Maps, 2016).

QUADRO 2. Descrição das amostras experimentais.

\begin{tabular}{cccc}
\hline Espécie & Nome científico & Nome vulgar & Espécie \\
A & Caesalpinia echinata & Pau-brasil & \\
B & Caesalpinia leiostachya & Pau-ferro & \\
C & Schizolobium parahyba & Guapuruvú & \\
D & Pterogyne nitens & Amendoim Bravo & \\
& & &
\end{tabular}




\section{RESULTADOS E DISCUSSÃO}

A tabela 1 apresenta os resultados relativos ao comportamento do crescimento, adensamento e adaptação das espécies florestais em lago artificial.

TABELA 1. Características biométricas das mudas mantidas em campo $(n=20$ indivíduos) no CTUR/UFRRJ, Seropédica-RJ, Brasil.

\begin{tabular}{|c|c|c|c|c|c|c|c|c|c|c|c|c|c|c|c|}
\hline \multirow{3}{*}{ Espécie } & \multicolumn{5}{|c|}{ Altura (cm) } & & \multirow{2}{*}{\multicolumn{4}{|c|}{$\begin{array}{c}\text { Diâmetro }(\mathrm{cm}) \\
\text { Período }\end{array}$}} & \multirow{2}{*}{\multicolumn{4}{|c|}{$\begin{array}{c}\text { Número Folhas } \\
\text { Período }\end{array}$}} & \multirow[b]{3}{*}{$\begin{array}{l}\text { Média } \\
\text { (unid.) }\end{array}$} \\
\hline & \multirow[b]{2}{*}{ TO } & \multicolumn{3}{|c|}{ Período } & \multirow[b]{2}{*}{$\begin{array}{l}\text { Média } \\
(\mathrm{cm})\end{array}$} & \multirow[b]{2}{*}{ TO } & & & & & & & & & \\
\hline & & T1 & T2 & T3 & & & & & T3 & $\begin{array}{l}\text { Média } \\
\text { (cm) }\end{array}$ & TO & T1 & & & \\
\hline$A_{1}$ & 150,0 & 151,0 & 154,0 & 160,0 & 154,5 & 3,0 & 4,0 & 4,0 & 5,0 & 4,0 & 12,0 & 12,0 & 11,0 & 15,0 & 12,5 \\
\hline$A_{2}$ & 87,0 & 3,0 & 93,0 & 100,0 & 91,0 & 3,0 & 4,0 & 4,0 & 4,0 & 3,8 & 5,0 & 5,0 & 7,0 & 39,0 & 14,0 \\
\hline$A_{3}$ & 100,0 & 108,0 & 108,5 & 109,0 & 106,3 & 4,0 & 4,5 & 4,5 & 4,5 & 4,4 & 7,0 & 8,0 & 8,0 & 10,0 & 8,3 \\
\hline $\mathbf{A}_{4}$ & 97,0 & & 113 & 110,0 & 108,3 & 5,0 & 5,0 & 5,0 & 5,0 & 5,0 & 8,0 & 8,0 & 11,0 & 12,0 & 9,8 \\
\hline $\begin{array}{c}\text { A } \\
\text { (Média) }\end{array}$ & 109,5 & 116,0 & 117,0 & 126,3 & 117,2 & 3,8 & 4,4 & 4,4 & 4,6 & 4,3 & 8,0 & 8,3 & 9,3 & 19,0 & 11,1 \\
\hline$B_{1}$ & 104,0 & 106,0 & 107,0 & 104,0 & 105,3 & 4,0 & 4,0 & 4,0 & 4,0 & 4,0 & 19,0 & 0,0 & 13,0 & 37,0 & 17,3 \\
\hline $\mathbf{B}_{2}$ & 92,0 & 0 & 97,0 & 86 & 92,5 & 2,0 & 2,0 & 3,0 & 3,0 & 2,5 & 9,0 & 14,0 & 2,0 & & \\
\hline $\mathbf{B}_{3}$ & 76,0 & 87 & 87,0 & 83 & 83,3 & 3,0 & 3,0 & 3,0 & 4,0 & 3,3 & 26,0 & 23,0 & 15,0 & & 25,0 \\
\hline $\mathrm{B}_{4}$ & 77,0 & 93,0 & 96,0 & 85 & 87,8 & 3,0 & 3,0 & 4,0 & 4,0 & 3,5 & 12,0 & 24,0 & 27,0 & 38,0 & 25,3 \\
\hline $\begin{array}{c}\text { B } \\
\text { (Média) }\end{array}$ & 87,3 & 95,3 & 96,8 & 89,5 & 92,2 & 3,0 & 3,0 & 3,5 & 3,8 & 3,3 & 16,5 & 15,3 & 14,3 & 35,3 & 20,3 \\
\hline $\mathrm{C}_{1}$ & 150,0 & 154,0 & 155,0 & 156,0 & 153,8 & 4,0 & 5,0 & 5,0 & 5,0 & 4,8 & 72,0 & 45,0 & 20,0 & 14,0 & 37,8 \\
\hline $\mathrm{C}_{2}$ & 65,0 & & 85,0 & 89,0 & 80,8 & 4,0 & 5,0 & 5,0 & 5,0 & 4,8 & 32,0 & 28,0 & 3,0 & 0 & 27,8 \\
\hline $\mathrm{C}_{3}$ & 116,0 & 118,0 & 119,0 & 121,0 & 118,5 & 4,0 & 5,0 & 5,0 & 5,0 & 4,8 & 23,0 & 14,0 & 15,0 & 0 & 25,3 \\
\hline $\mathrm{C}_{4}$ & 72,0 & 020 & 020 & & $00-$ & 4,0 & 4,0 & 4,0 & 5,0 & 4,3 & 13,0 & 3,0 & 22,0 & 19,0 & 14,3 \\
\hline $\begin{array}{c}\text { C } \\
\text { (Média) }\end{array}$ & 100,8 & 109,5 & 110,5 & 112,8 & 108,4 & 3,8 & 4,4 & 4,5 & 4,8 & 4,4 & 35,0 & 22,5 & 20,0 & 27,5 & 26,3 \\
\hline$D_{1}$ & 92,0 & & 98,0 & 103,0 & 97,0 & 4,0 & 5,0 & 5,0 & 5,0 & 4,8 & 9,0 & 3,0 & 2,0 & 37,0 & 12,8 \\
\hline$D_{2}$ & 58,0 & & 62,0 & & & 4,0 & 4,0 & 4,0 & 4,0 & 4,0 & 4,0 & 3,0 & 3,0 & 7,0 & 4,3 \\
\hline $\mathrm{D}_{3}$ & 109,0 & 120,0 & 123,0 & 138 & 122,5 & 5,0 & 6,0 & 6,0 & 6,0 & 5,8 & 4,0 & 4,0 & 4,0 & 33,0 & 11,3 \\
\hline $\mathrm{D}_{4}$ & 90,0 & 94,0 & 98,0 & 101,0 & 95,8 & 3,0 & 4,5 & 4,5 & 4,8 & 4,2 & 6,0 & 5,0 & 5,0 & 6,0 & 5,5 \\
\hline $\begin{array}{c}\text { D } \\
\text { (média) }\end{array}$ & 87,3 & 92,3 & 95,3 & 101,0 & 93,9 & 4,0 & 4,9 & 4,9 & 4,9 & 4,7 & 5,8 & 3,8 & 3,5 & 20,8 & 8,4 \\
\hline$E_{1}$ & 54,0 & 56,0 & 59,0 & 68,0 & 59,3 & 2,0 & 3,0 & 3,0 & 3,0 & 2,8 & 8,0 & 6,0 & 13,0 & 19,0 & 11,5 \\
\hline$E_{2}$ & 78,0 & 85,0 & 89,0 & 92 & 86,0 & 3,0 & 3,0 & 4,0 & 4,0 & 3,5 & 4,0 & 6,0 & 7,0 & 7,0 & 6,0 \\
\hline$E_{3}$ & 66,0 & 78,0 & 80,0 & 84 & 77,0 & 2,0 & 3,0 & 4,0 & 4,0 & 3,3 & 4,0 & 5,0 & 10,0 & 23,0 & 10,5 \\
\hline$E_{4}$ & 50,0 & 60,0 & 61,0 & 03,0 & 58,5 & 2,5 & 3,0 & 3,0 & 3,0 & 2,9 & 8,0 & 8,0 & 8,0 & 39,0 & 15,8 \\
\hline $\begin{array}{c}E \\
\text { (Média) }\end{array}$ & 62,0 & 69,8 & 72,3 & 76,8 & 70,2 & 2,4 & 3,0 & 3,5 & 3,5 & 3,1 & 6,0 & 6,3 & 9,5 & 22,0 & 10,9 \\
\hline
\end{tabular}

Legenda: $A=$ Caesalpinia echinata (Pau-brasil); $B=$ Caesalpinia leiostachya (Pauferro); $C=$ Schizolobium parahyba (Guapuruvú); $D=$ Pterogyne nitens (Amendoim Bravo); $E=$ Tabebuia chrysotricha (1pê-amarelo); $T 0=$ tempo inicial; $T 1=$ após 30 dias; $T 2=$ após 60 dias; $T 3=$ após 90 dias.

A Figura 3 apresenta média do crescimento das espécies da altura $(\mathrm{cm})$ em função do tempo (0,30, 60 e 90 dias), sendo observados os seguintes valores: 126,3 cm para Caesalpinia echinata (Pau-brasil); $112,8 \mathrm{~cm}$ na Schizolobium parahyba (Guapuruvú); 101,0 cm no Pterogyne nitens (Amendoim Bravo); 98,2 para Caesalpinia leiostachya (Pau-ferro); e 76,8 cm na Tabebuia chrysotricha (Ipêamarelo). 


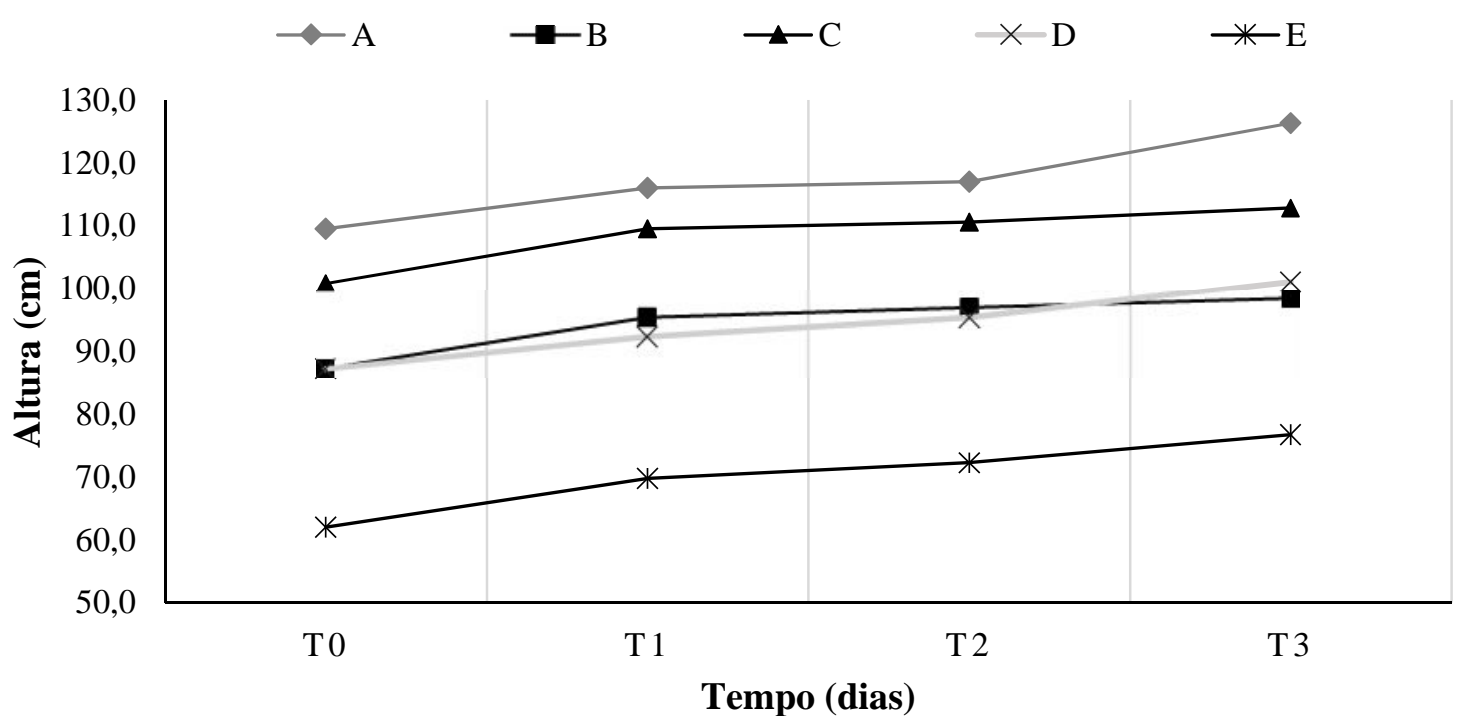

FIGURA 3 - Crescimento em altura $(\mathrm{cm})$ das espécies $A=$ Caesalpinia echinata (Pau-brasil); $B=$ Caesalpinia leiostachya (Pau-ferro); $C=$ Schizolobium parahyba (Guapuruvú); $D=$ Pterogyne nitens (Amendoim Bravo); $E=$ Tabebuia chrysotricha (Ipê-amarelo); TO= tempo inicial; $T 1=$ após 30 dias; $T 2=$ após 60 dias; $T 3=$ após 90 dias.

Apesar das espécies de estágios sucessionais iniciais apresentarem tendência de maior crescimento em altura para espaçamento adotado como desse estudo, a Caesalpinia echinata (Pau-brasil) apresentou maior crescimento nos 90 dias de investigação. A espécie Caesalpinia echinata (Pau-brasil), apresentou comportamento diferente das observações de Nascimento et al. (2013), as plantas das espécies pioneiras mostram maior resposta de crescimento ao espaçamento menos adensado.

A Figura 4 exibe o comportamento do parâmetro biométrico do diâmetro do caule $(\mathrm{cm})$ no período observado, os valores foram de $4,9 \mathrm{~cm}$ para o Pterogyne nitens (Amendoim Bravo); em seguida a espécie Schizolobium parahyba (Guapuruvú) com 4,8 cm; posteriormente a Caesalpinia echinata (Pau-brasil) apresentou 4,6 cm; o Caesalpinia leiostachya (Pau-ferro) com 3,8 cm; e pôr fim a espécie Tabebuia chrysotricha (lpê-amarelo) produziu 3,5 cm.

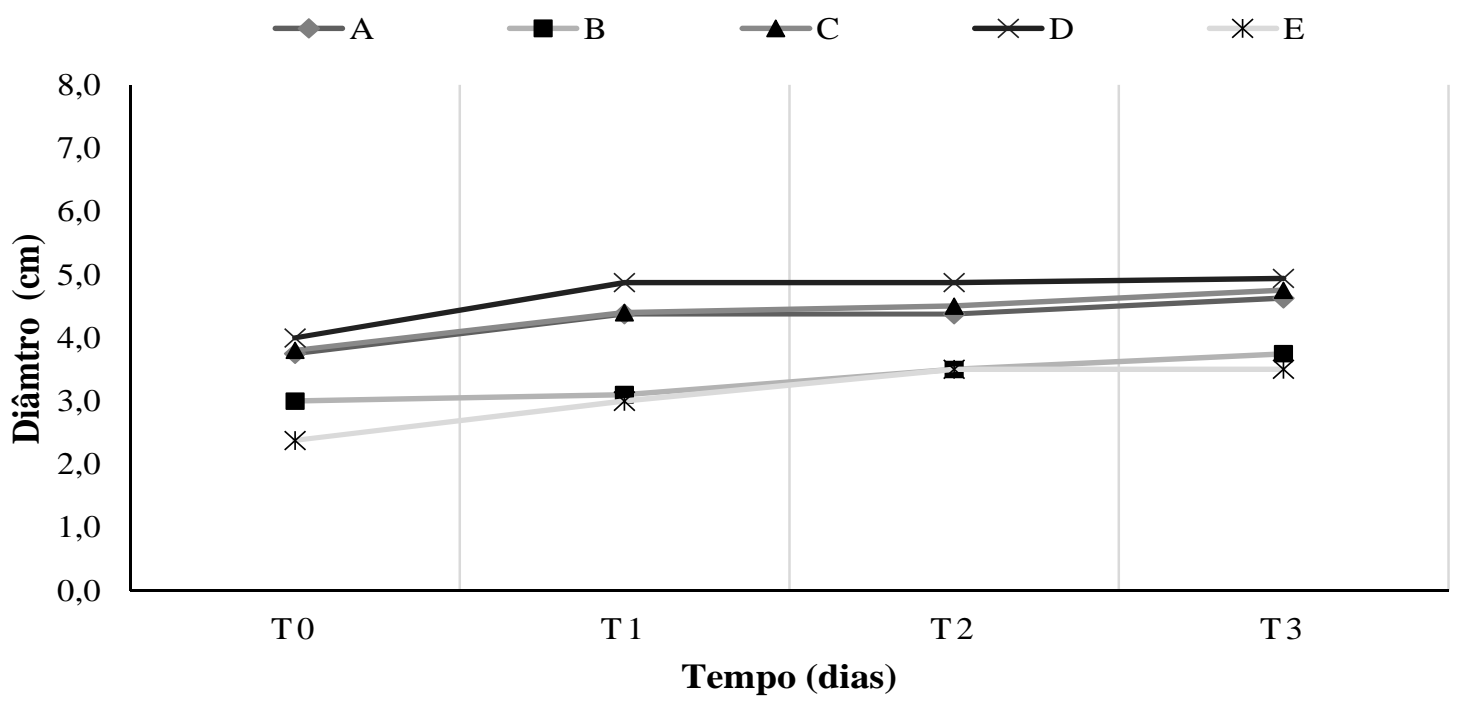

FIGURA 4. Crescimento em diâmetro do caule $(\mathrm{cm})$ das espécies $A=$ Caesalpinia echinata (Paubrasil); $B=$ Caesalpinia leiostachya (Pau-ferro); $C=$ Schizolobium parahyba (Guapuruvú); $\mathrm{D}=$ Pterogyne nitens (Amendoim Bravo); $E=$ Tabebuia chrysotricha (Ipêamarelo); $T 0=$ tempo inicial; $T 1=$ após 30 dias; $T 2=$ após 60 dias; $T 3=$ após 90 dias. 
O pouco adensamento do caule apresentou pouca diferença entre as espécies, o qual pode estar associada a baixa competição intraespecífica no espaçamento, como relatado na literatura por Daniel et al. (1982). A Figura 5 apresenta análise da média no número de folhas das espécies investigadas ao logo dos 90 dias, os valores encontrados foram: 35,3 para Caesalpinia leiostachya (Pauferro); 27,5 para Schizolobium parahyba (Guapuruvú); 22,0 para Tabebuia chrysotricha (Ipê-amarelo); 20,8 no Pterogyne nitens (Amendoim Bravo); e 19,0 para Caesalpinia echinata (Pau-brasil).

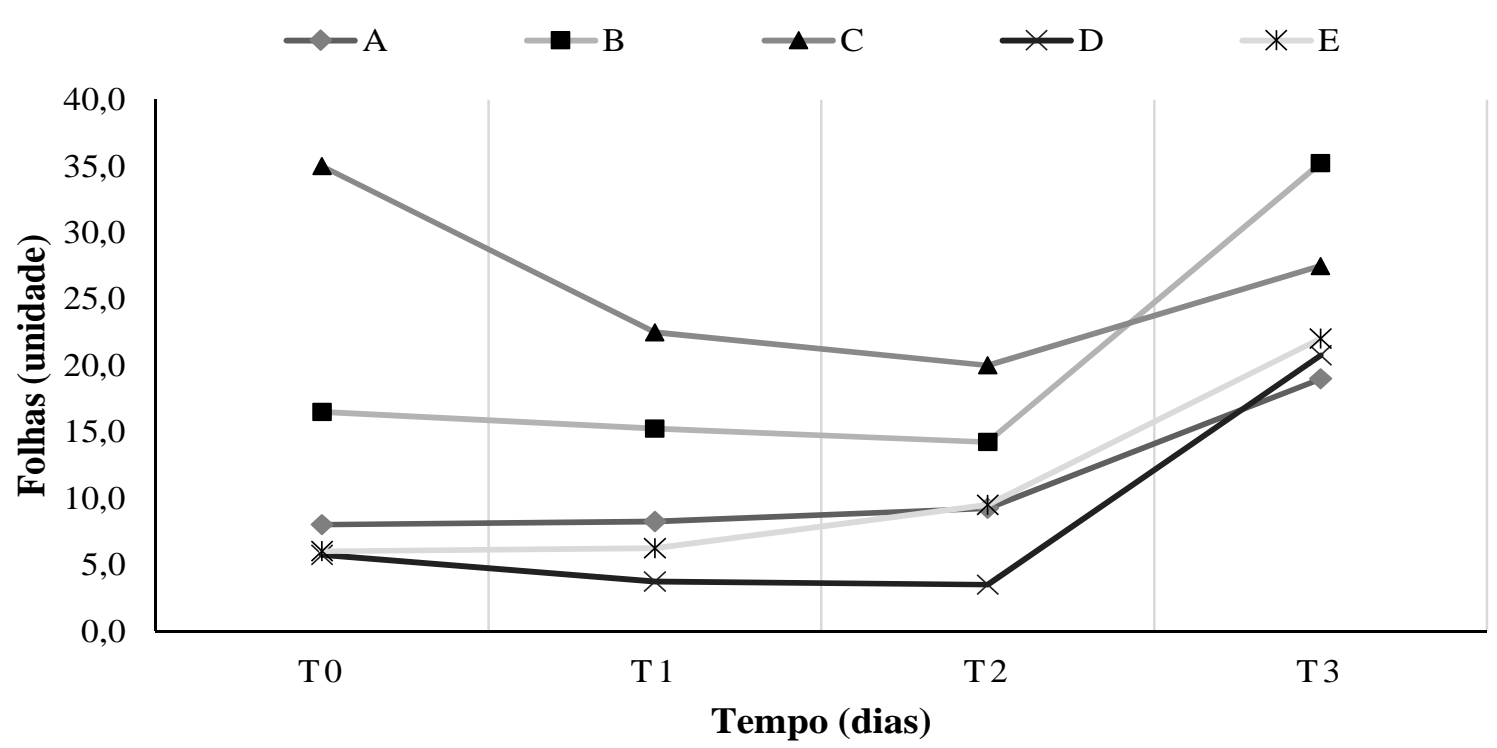

FIGURA 5. Número de folhas das espécies $A=$ Caesalpinia echinata (Pau-brasil); $B=$ Caesalpinia leiostachya (Pau-ferro); $C=$ Schizolobium parahyba (Guapuruvú); $\mathrm{D}=$ Pterogyne nitens (Amendoim Bravo); $E=$ Tabebuia chrysotricha (Ipê-amarelo); $T 0=$ tempo inicial; $T 1=$ após 30 dias; $T 2=$ após 60 dias; $T 3=$ após 90 dias.

Todas as espécies apresentaram aumento de folhas após 90 dias de plantio, porém durante 0 intervalo de 0 a 60 dias, houve queda nesse parâmetro para estas espécies, exceto a espécie Schizolobium parahyba (Guapuruvú). A queda do número de folhas até 60 dias (Figura 5 ) de observação de todas espécies analisadas, demostra que essas sofreram stress as condições edafoclimáticas após realizado plantio no campo, e recuperação e adaptação até 90 dias de observação, conforme Leles et al. (2011).

\section{CONCLUSÃO}

Diante do trabalho realizado, verificou-se que espécie Caesalpinia echinata (Pau Brasil) apresentou desempenho satisfatório em relação às demais, quanto ao crescimento em altura (cm), e na Tabebuia chrysotricha (Ipê-amarelo) foi observado menor valor.

Em relação ao crescimento do caule $(\mathrm{cm})$ os valores oscilaram em $4,9 \mathrm{~cm}$ para o Pterogyne nitens (Amendoim Bravo) e 3,5 cm para Tabebuia chrysotricha (Ipê-amarelo).

Contudo, recomenda-se a continuidade do monitoramento e manutenção do plantio em até 2 anos, bem como o enriquecimento com outras espécies florestais. 


\section{AGRADECIMENTOS}

Ao Jardim Botânico e Instituto de Florestas da UFRRJ pela doação das mudas, e aos 52 colegas do curso Técnico em Meio Ambiente do CTUR/UFRRJ pelo apoio operacional, ao Sr. Hugo Neves pelo apoio técnico, aos funcionários de Campo pelo empenho, bem como a Direção do CTUR/UFRRJ pelo apoio institucional.

\section{REFERÊNCIAS}

ALARCON, G.G.; AYANU, Y.; FANTINI, A.C.; FARLEY, J.; SCHMITT FILHO, A.; KOELLNER, T. Weakening the Brazilian legislation for forest conservation has severe impacts for ecosystem services in the Atlantic Southern Forest. Land Use

Policy, v. 47, p. 1-11, 2015. Disponível em: https://www.sciencedirect.com/science/article/pii/S0264837715000848. DOI: 10.1016/j.landusepol.2015.03.011.

BRASIL, República Federativa. Lei Federal n. 12.651, de 25 de maio de 2012. Dispõe sobre a proteção da vegetação nativa; altera as Leis ns. ${ }^{\circ} 6.938 / 1981$ e 9.393/1996; revoga as Leis nos 4.771/1965 e 7.754/1989. Brasília: Diário Oficial [da] República Federativa do Brasil. 28 de Mai. de 2012.

FARIAS, R.C.; LACERDA, A.V.; GOMES, A.C.; BARBOSA, F.M.; MAIA, C.S. Riqueza florística em uma área ciliar de Caatinga no Cariri Ocidental da Paraíba, Brasil. Revista Brasileira de Gestão Ambiental e Sustentabilidade, v. 4, n. 7, p. 109-118, $2017 . \quad$ Disponível em: http://revista.ecogestaobrasil.net/v4n7/v04n07a11.pdf. DOI: 10.21438/rbgas.040711.

FREITAS, E.P.; MORAES, J.F.; PECHE FILHO, A.; STORINO, M. Indicadores ambientais para áreas de preservação permanente. Revista Brasileira de Engenharia Agrícola e Ambiental, v. 17, n. 4, p. 443-449, 2013. Disponível em: http://www.scielo.br/scielo.php?pid=S1415-

43662013000400013\&script=sci_abstract\&tlng=pt. $\quad$ DOI: $\quad 10.1590 / S 1415-$ 43662013000400013.

FUNDAÇÃO SOS MATA ATLÂNTICA; INSTITUTO NACIONAL DE PESQUISAS ESPACIAIS (INPE). Atlas dos remanescentes florestais da Mata Atlântica período 2014-2015: relatório técnico. São Paulo: Fundação SOS Mata Atlântica \& Instituto Nacional de Pesquisas Espaciais, 2016. Disponível em: https://www.sosma.org.br/105066/mg-volta-liderar-ranking-de-desmatamento-damata-atlantica/. Acessado em: 27 de Jul. de 2016.

\section{GOOGLE MAPS. Imagem do Colégio Técnico da Universidade Federal Rural do Rio de Janeiro. Disponível em:} https://www.google.com.br/maps/place/Serop\%C3\%A9dica+-+RJ/@-22.7511845,43.7102944,11z/data=!3m1!4b1!4m2!3m1!1s0x995679694622cb:0x87984001ac8db $1 \mathrm{f} 2$ ?hl=pt-BR. Acessado em: 10 abr. 2016.

LEITE, T. D.S.; DOMBROSKI, J.L.D.; FREITAS, R.M.O.D.; LEITE, M.D.S.; RODRIGUES, M.R.D.O. Production of Enterolobium Contortisiliquum Seedlings and Assimilate Partitioning In Response To Phosphorus Fertilization And Inoculation With Mycorrhizal Fungi. Ciência Florestal, v. 27, n. 4, p. 1157-1166, 2017. 
LELES, P.S.S.; ABAURRE, G.W.; ALONSO, J.M.; NASCIMENTO, D.F.; LISBOA, A. Crescimento de espécies arbóreas sob diferentes espaçamentos em plantio de recomposição florestal. Scientia Forestalis, Piracicaba, v. 39, n. 90, p. 231-239, 2011.

NASCIMENTO, D.F.; LELES, P.S.S.; OLIVEIRA NETO, S.N.; MOREIRA, R.T.S.; MAKHLOUTA, A.J. Crescimento inicial de seis espécies florestais em diferentes espaçamentos. Cerne, v. 18, n. 1, p.159-165 2013.

RIBEIRO, M.; RODRIGUES, R.R.; PAESE, A.; COSTA, S.; HENRIQUE, P.; BRANCALION, S.; SANTIAMI, E.; PEREIRA, G.; GUIMARÃES, J.; KOCK, R. A restauração da Mata Atlântica apoiada em sistemas de informações geográficas. In.: PAESE, A., UEZU, A., LORINI, M.L.; CUNHA, A. (orgs.). Conservação da biodiversidade com SIG. São Paulo: Oficina de Textos, 2015. p. 32-54.

RODRIGUES, L.G.S.M.; GOI, S.R.; RODRIGUES, F.M. Associação micorrízica como uma estratégia para o estabelecimento de espécies em áreas impactadas. Journal of Bioenergy and Food Science, v.1, n.1, p.7-16, 2014.

RONDON, E.V. Produção de biomassa e crescimento de árvores de Schizolobium amazonicum (Hub.) Ducke sob diferentes espaçamentos na região de mata. Revista Árvore, v. 26, n. 5, p. 573-576, 2002.

SILVA, B.M. Avaliação do Projeto de Revegetação de Área Degradada na margem do Rio Paraíba do Sul: avaliação dos primeiros resultados. Boletim do Observatório Ambiental Alberto Ribeiro Lamego, v. 2, n. 1, p. 59-78, 2008. 